\title{
Bilateral symmetry of vision disorders in typical retinitis pigmentosa
}

\author{
ROBERT W. MASSOF, DANIEL FINKELSTEIN, STUART J. STARR, \\ KENNETH R. KENYON, JAY A. FLEISCHMAN, AND IRENE H. MAUMENEE \\ From the Wilmer Ophthalmological Institute, Johns Hopkins University School of Medicine, Baltimore, \\ Maryland, USA
}

SUMMARY Bilateral symmetry of disorders of vision is examined in 60 typical patients with retinitis pigmentosa. We observed a very high degree of interocular congruence in the patterns of both kinetic visual field defects and threshold profiles and in abnormalities of foveal colour discrimination and visual acuity. Abnormalities of foveal colour vision are highly correlated with the extent of visual field loss.

Typical retinitis pigmentosa (RP) is a clinical syndrome of inherited diffuse pigmentary retinal dystrophy characterised by (1) nyctalopia, (2) midperipheral ring-like scotoma or field contraction, (3) narrowed retinal arterioles, (4) mid-peripheral intraretinal 'bone-spicule' pigmentation, (5) relatively good visual acuity until late in the course of the disease, and (6) no known toxic, systemic metabolic, or inflammatory origin of the degeneration. The various modes of inheritance of typical RP have been identified as autosomal dominant, autosomal recessive, X-linked, and sporadic, usually assumed to be recessive in origin (Jay, 1972). It is a general clinical impression that progression of the disease is bilaterally symmetrical (Pearlman, 1977), although exceptions to this symmetry have been epitomised in cases described as 'unilateral retinitis pigmentosa' (Carr and Siegal, 1973).

The documentation of bilateral symmetry has received little formal attention. Gonin (1901), in describing the ring scotoma as a characteristic feature of typical RP, concluded from 6 of his own cases and a review of 20 other cases that the ring scotoma was 'bilateral and symmetric'. Zeavin and Wald (1956) noted a strong bilateral symmetry in absolute threshold profiles measured in $6 \mathrm{RP}$ patients. Biro (1963), briefly summarising observations of fundus pigmentation in over 500 cases, concluded that the 'tendency to symmetrical de-

Address for reprints: Robert W. Massof, PhD, Wilmer Institute, B-34, Johns Hopkins University School of Medicine, Baltimore, Maryland 21205 , USA velopment (of intraretinal pigmentation) is extremely characteristic'.

The present study examines bilaterial symmetry of vision disorders in 60 typical RP patients. We observed a very high degree of bilateral similarity in the pattern of kinetic visual field defects, absolute threshold profiles, and abnormalities in foveal colour discrimination and visual acuity. Furthermore, the present study finds that the abnormalities of foveal colour vision are highly correlated with the extent of visual field loss.

\section{Patients and methods}

We evaluated 60 patients meeting our 6 criteria of typical RP (outlined above). Patients ranged in age from 8 to 70 years. The modes of inheritance were distributed as follows: $33 \%$ autosomal dominant, $4 \% \mathrm{X}$-linked, and $63 \%$ autosomal recessive or sporadic. Informed consent was obtained from each patient participating in the study.

All tests were performed monocularly on each eye of each patient. After correction of refractive error the visual acuity was determined. Kinetic visual fields were measured with a Goldmann perimeter using the $\mathrm{V} / 4$ target. Colour discrimination was measured with the Farnsworth-Munsell 100 hue test using a Macbeth illuminant.

Absolute thresholds were measured at the fovea and at 7 to 11 peripheral positions along each of 4 semimeridians (nasal, temporal, superior, and inferior) employing the Tübinger perimeter (Sloan, 1971). Threshold values were obtained for a blue- 
Fig. 1 Log decimal visual acuity of right eye is plotted against log decimal visual acuity of left eye for each patient (points). Snellen notation is also indicated on the figure. The dotted lines bound a range of $\pm 0 \cdot 3 \log$ units (approximately \pm 1 line on the visual acuity (hart)

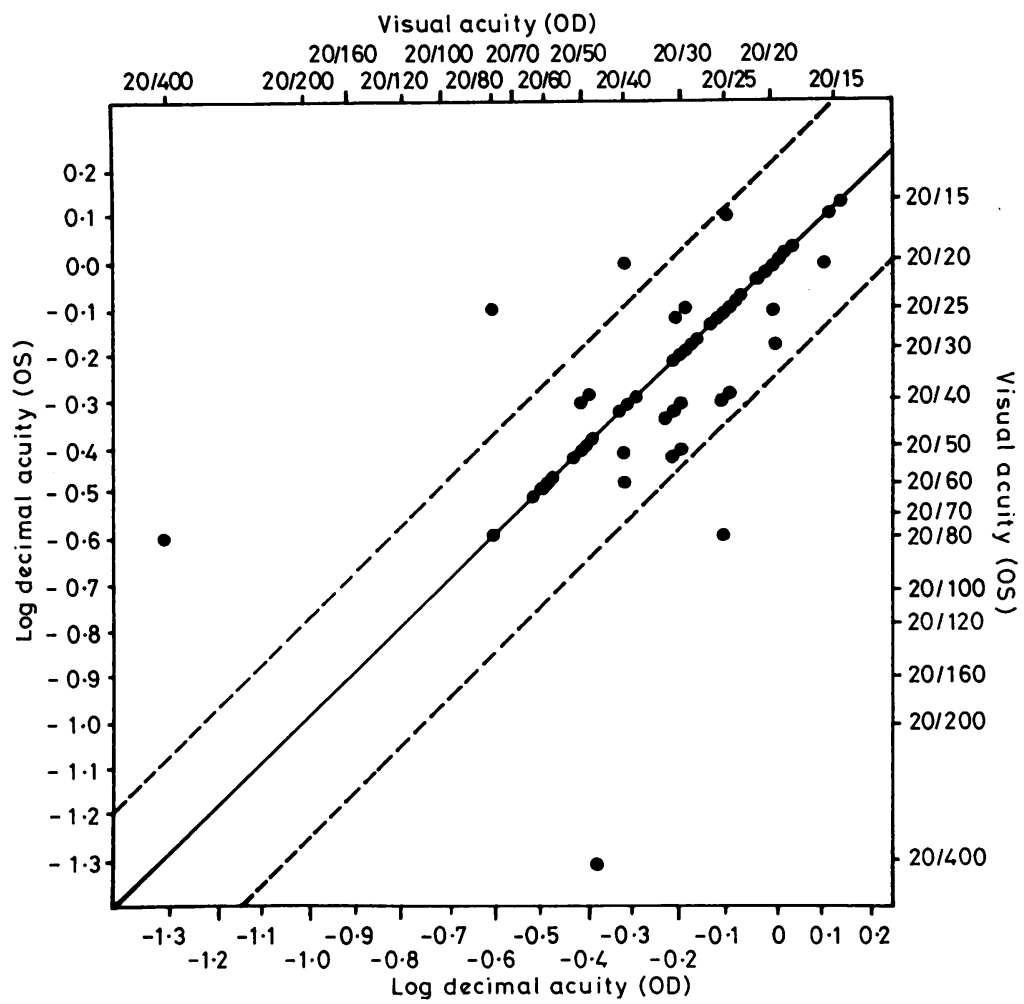

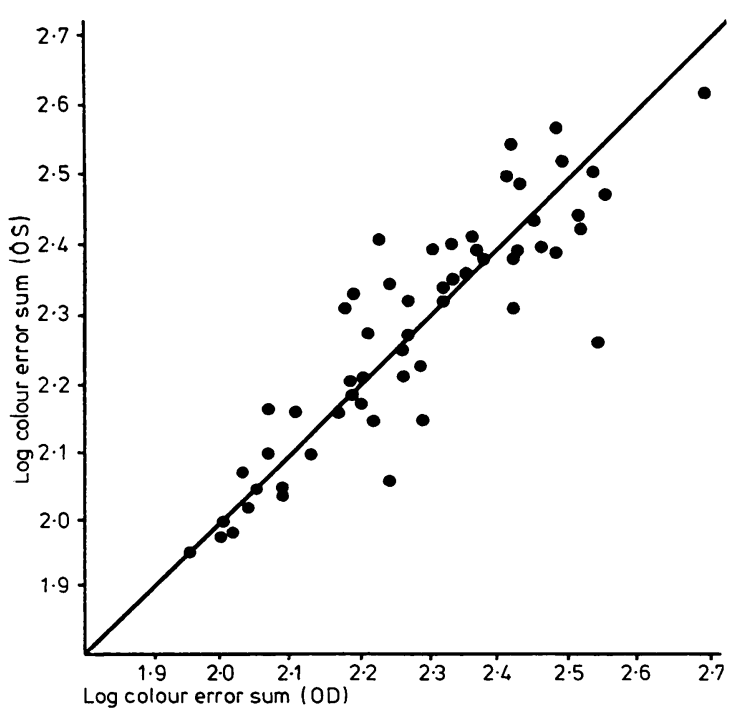

Fig. 2 Log colour error sum for the right eye is plotted against the log colour error sum for the left eye for each patient (points) green light $\left(\lambda_{\max }=500 \mathrm{~nm}\right)$, subtending a visual angle of $2^{\circ}$, and flashed on a dark background for 500 milliseconds. The patient was dark-adapted for 45 minutes prior to the threshold measurements. Absolute thresholds were defined as the mean of 3 to 5 repetitions of an ascending method of limits (from below threshold) and were expressed as log relative values (maximum luminance $=2 \cdot 1 \mathrm{fL}$ ).

\section{Results}

VISUAL ACUITY

Visual acuity fractions were converted to log decimal acuity.* In Fig. 1 log decimal visual acuity for the right eye is plotted against the log decimal visual acuity for the left eye for each patient. If perfect bilateral symmetry were obtained, all the data points in Fig. 1 would fall on the solid diagonal line, that is, a perfect correlation between the 2 eyes would exist. To determine the extent of correlation between the visual acuities of the right and left eye a product moment correlation coefficient was calculated. A high correlation was obtained $(r=0.63$;

*Each line of the visual acuity chart represents approximately equal changes in angular subtense on a logarithmic scale. 

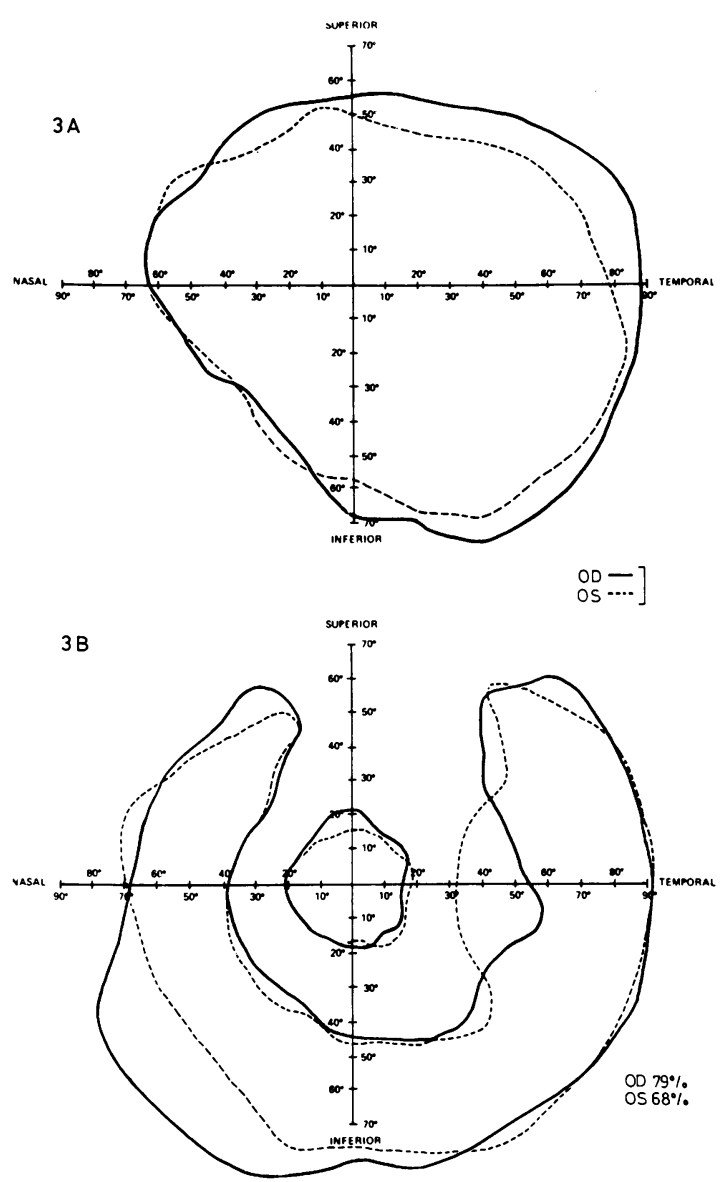

Figs $3 a, 3 b$

Fig. 3 Representative examples of visual fields from $(A)$ a normal observer and $(B-J)$ retinitis pigmentosa patients for the right eye (solid line) and left eye (dashed line), employing the V/4 target on the Goldmann perimeter. Visual fields for both eyes are plotted with respect to nasal and temporal orientation to compare patterns of field loss. The lines bound areas in which the target can be seen. The percentage of normal visual field area is indicated for each pair of fields

a perfect correlation is $r=1.00$ ), indicating that visual acuity in the right eye parallels visual acuity in the left eye.

\section{COLOUR DISCRIMINATION}

The results of the Farnsworth-Munsell 100 hue test were converted to a single error score by taking the
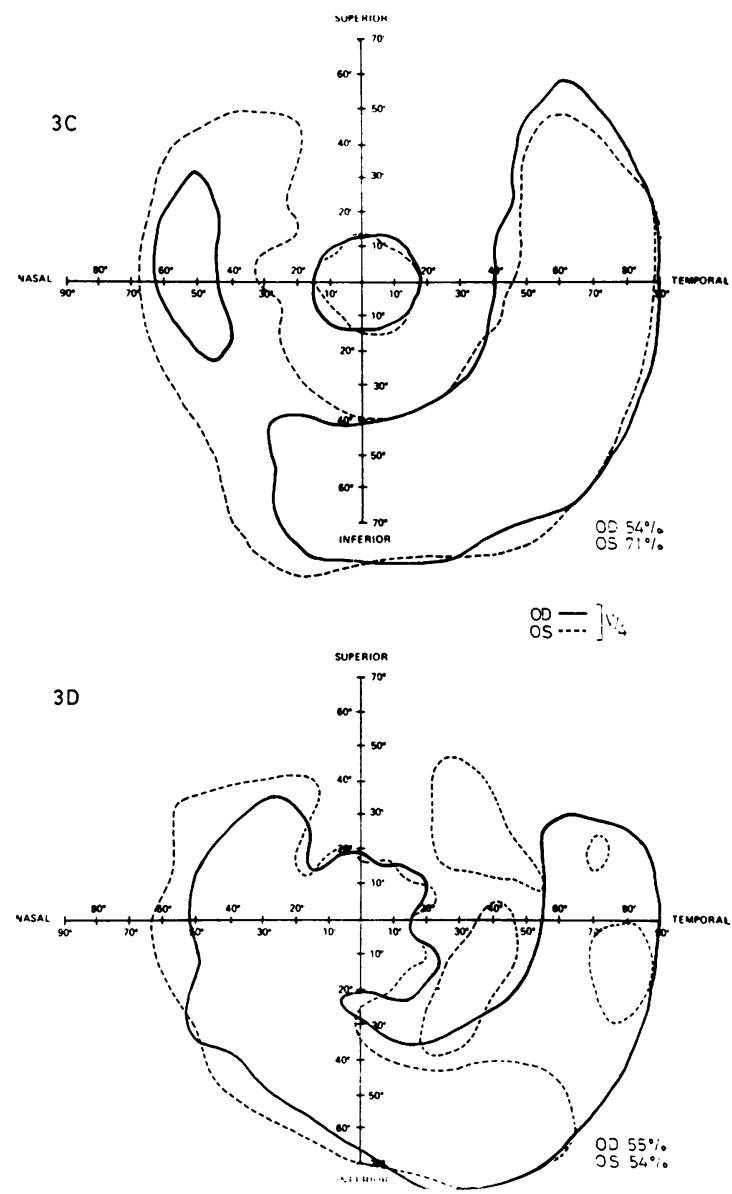

Figs $3 c, 3 d$

$\log$ of the sum of all errors. $\dagger$ In Fig. 2, the error score for the left eye is plotted against the error score for the right eye. If perfect symmetry were obtained, all the data points would fall on the diagonal line. Calculation of the product moment correlation yielded a very high coefficient $(r=0.88)$, indicating that loss of colour discrimination in the right eye parallels the loss in the left eye.

\section{VISUAL FIELDS}

A qualitative assessment of bilaterial symmetry in the pattern of visual field loss was made by plotting the mirror image of the visual field for the left eye over the visual field for the right eye. Fig. 3 illustrates representative examples of these comparisons.

tThe log of the error sums is employed because the variability in the error sums is proportional to the mean error sum. 

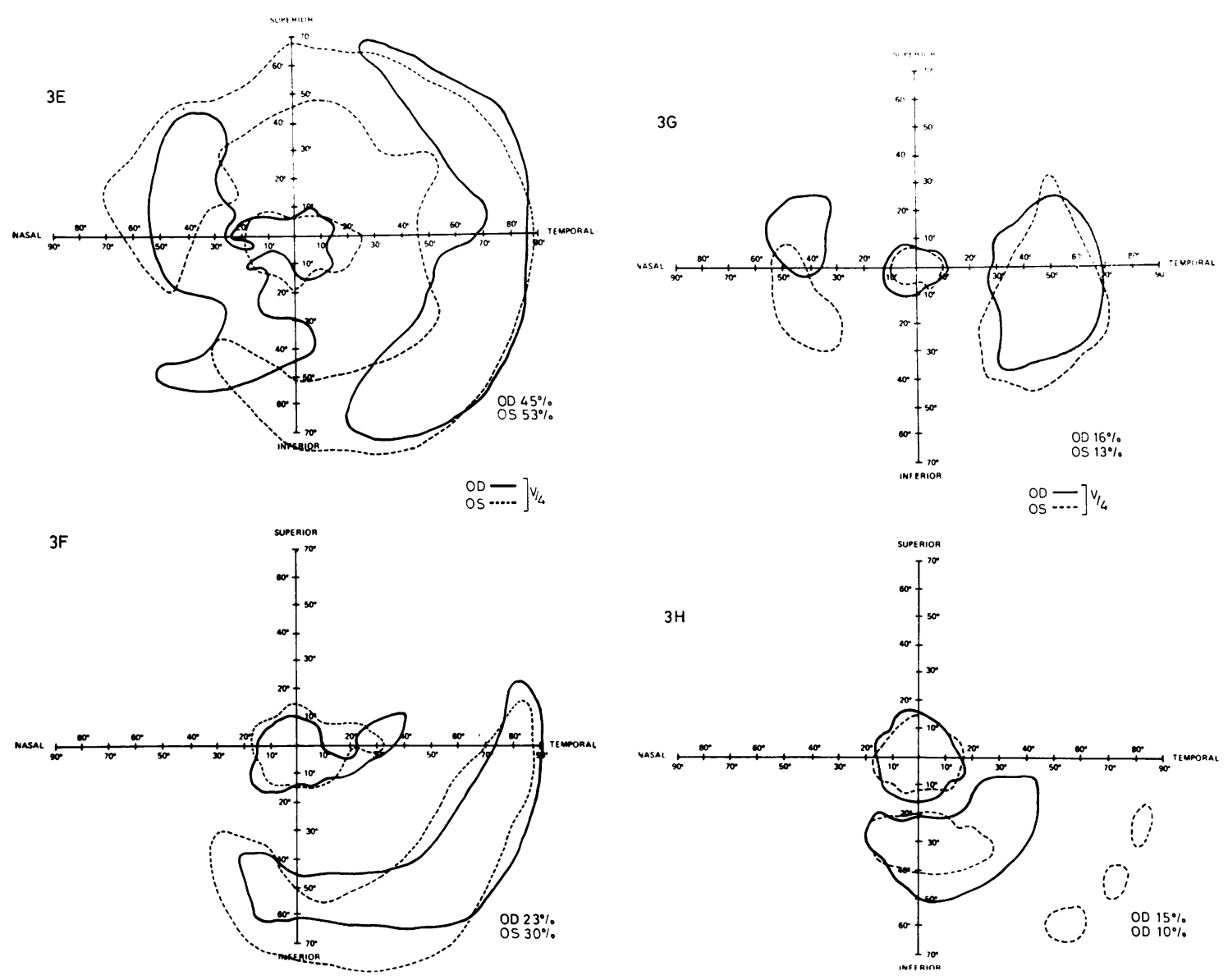

Figs $3 e, 3 f$

Figs $3 \mathrm{~g}, \mathbf{3 h}$

Each visual field was converted to a single number by measuring the total area of visual function with a planimeter. The resulting visual field area is expressed as a percentage of normal by dividing each value by the area of the average normal visual field. The numbers beside the visual fields in Fig. 3 indicate the measured areas of visual function (expressed as percentage of normal) for each example.

The numerical value for visual field area of the right eye is plotted against the numerical value for visual field area of the left eye, for each patient, in Fig. 4. If perfect bilateral symmetry were obtained, all the data points would fall on the diagonal line. The calculation of the product moment correlation yielded a nearly perfect correlation coefficient $(r=0.96)$. Thus, visual field area loss in the right eye parallels to a high degree the visual field area loss in the left eye.

\section{ABSOLUTE THRESHOLD PROFILES}

The mirror image of the sensitivity profile for the left eye was plotted along with the sensitivity profile for the right eye for each patient. As exemplified in Fig. 5, not only does the loss of sensitivity occur to about the same degree in each eye, but also the shapes of the sensitivity profiles are quite similar. These data complement the visual field data plotted in Fig. 3. A comparison of the data from patients with RP with normal values (Fig. 5L) demonstrates a 1.0 to $2.0 \mathrm{log}$ unit loss in foveal sensitivity for all patients.

\section{Discussion}

We found a high degree of bilaterial symmetry in loss of visual function in patients meeting the criteria of typical RP, independent of the mode of 


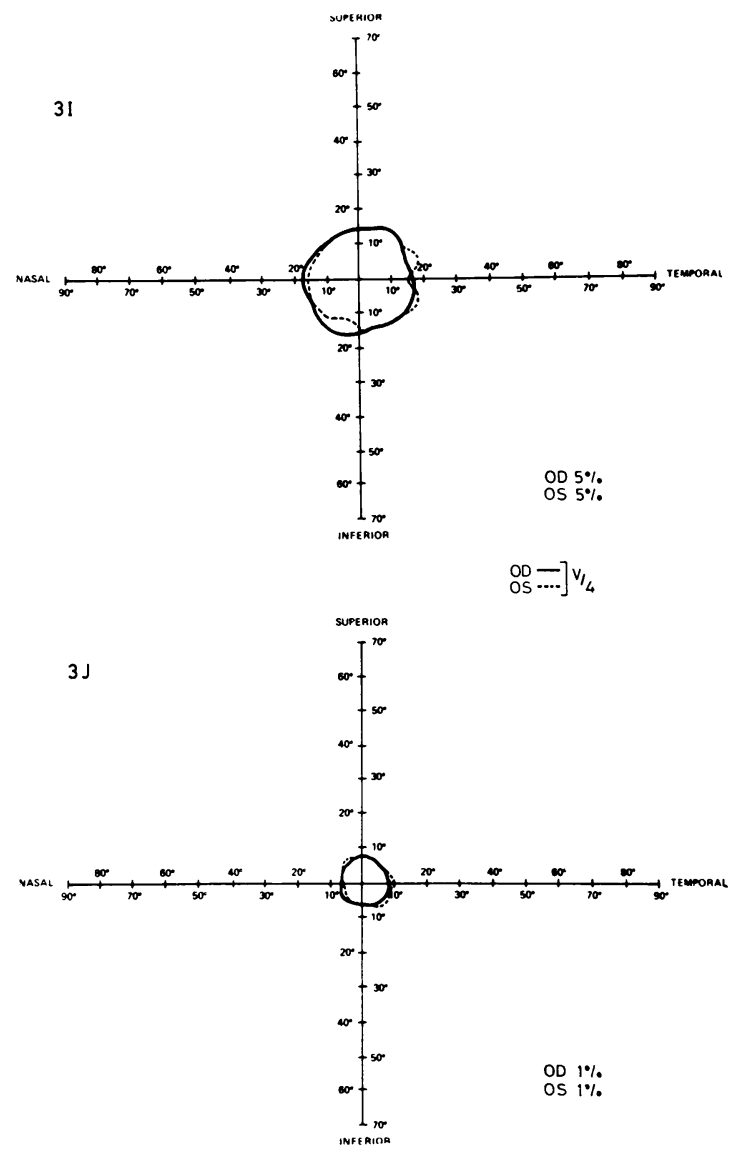

Figs $3 i, 3 i$

inheritance. This statement holds true for visual acuity, colour discrimination, kinetic visual fields, and static absolute threshold profiles. Such bilateral symmetry, in both the extent and pattern of loss of visual function, complemented by Biro's (1963) finding of symmetry in fundus pigmentation, suggests a high degree of congruence in the pattern and natural history of the retinal degeneration.

The finding of a high degree of symmetry in RP is important from the standpoint of evaluating therapy which employs one eye as a control (for example, Berson's (1971) total light exclusion trial) and for better understanding the natural course of RP. The symmetry in the pattern of vision loss (see Figs. 3 and 5) suggests that the progression follows morphological features yet to be identified. We are at present examining this suggestion in more detail.

A consistent observation in the present study was

Fig. 4 Visual field area expressed as a percentage of the normal visual field area for the right eye is plotted against the visual field area for the left eye for each patient (points)

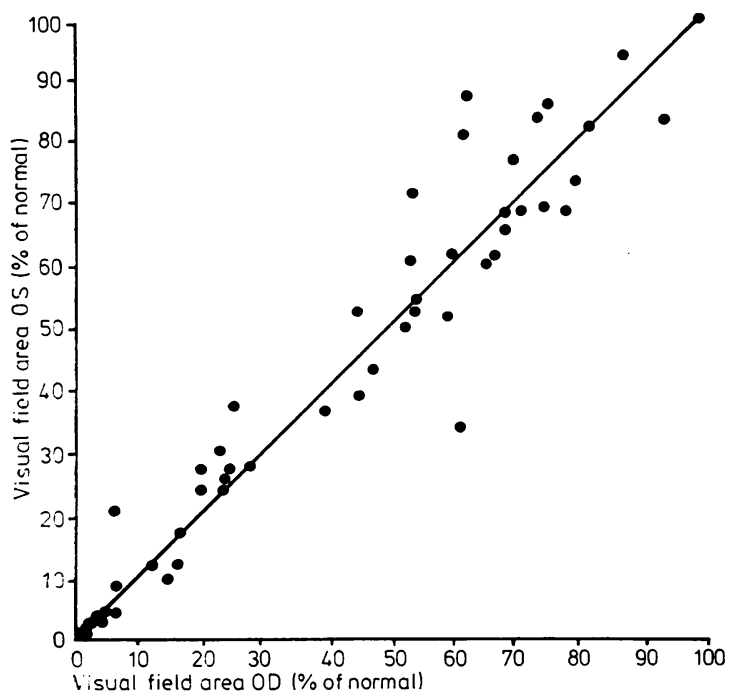

the presence of disorders of foveal vision at all stages of RP progression, as evident in the measures of colour discrimination and absolute foveal thresholds. Previous investigators have made similar observations (Zeavin and Wald, 1956; Sunga and Sloan, 1967; Adams et al., 1972), but such foveal abnormalities have been overshadowed by the usual persistence of good visual acuity until late in the course of RP. As shown in Fig. 6, we observed a high correlation between the colour error score and reduction in the area of visual field $(r=0.73)$. These results indicate that progression in loss of foveal colour discrimination is parallel to the loss of visual field.

In conclusion, we have found that bilateral symmetry in loss of visual function is a characteristic feature of typical RP. Also, from the high degree of congruence in visual fields and threshold profiles, 


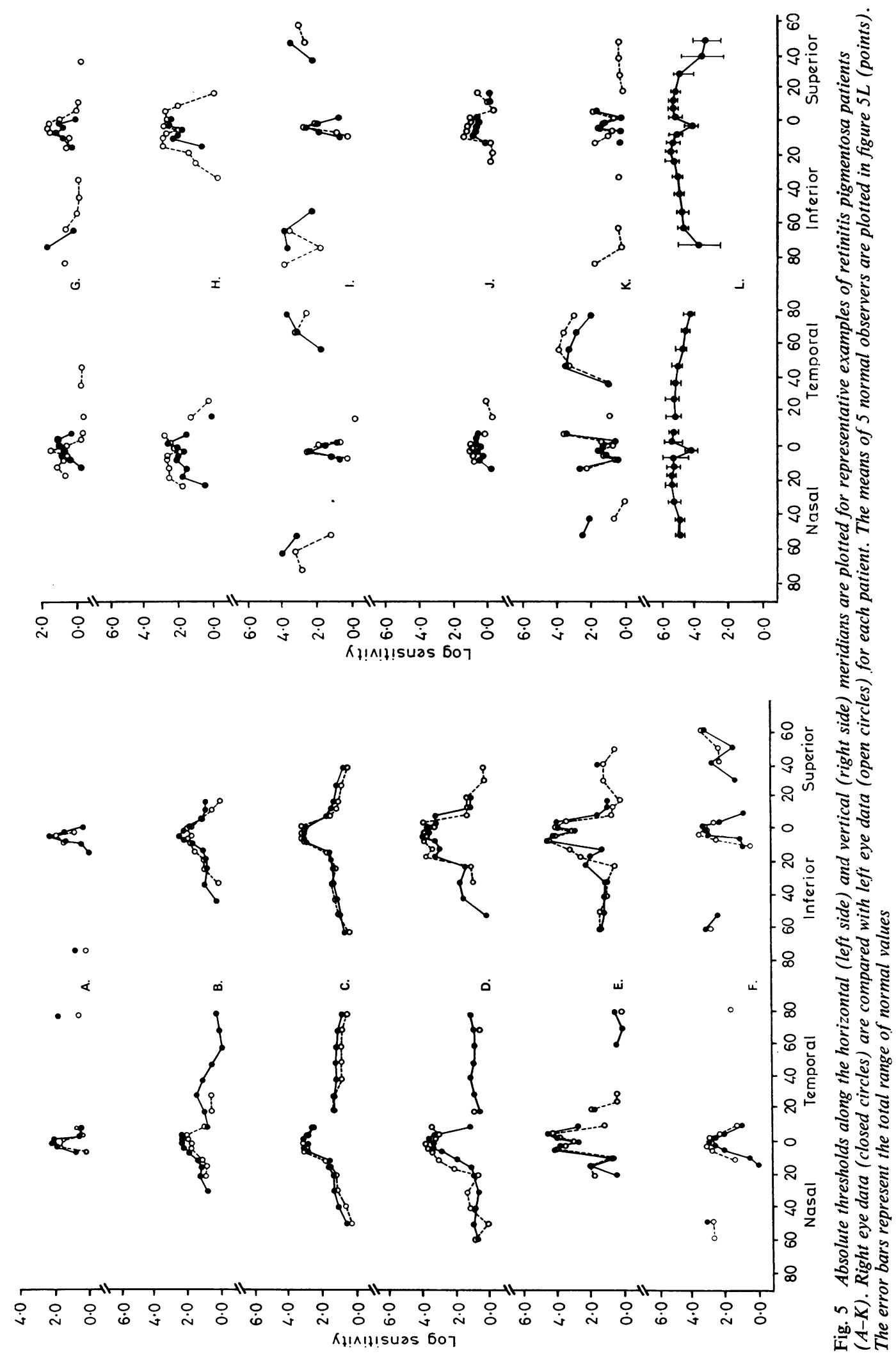




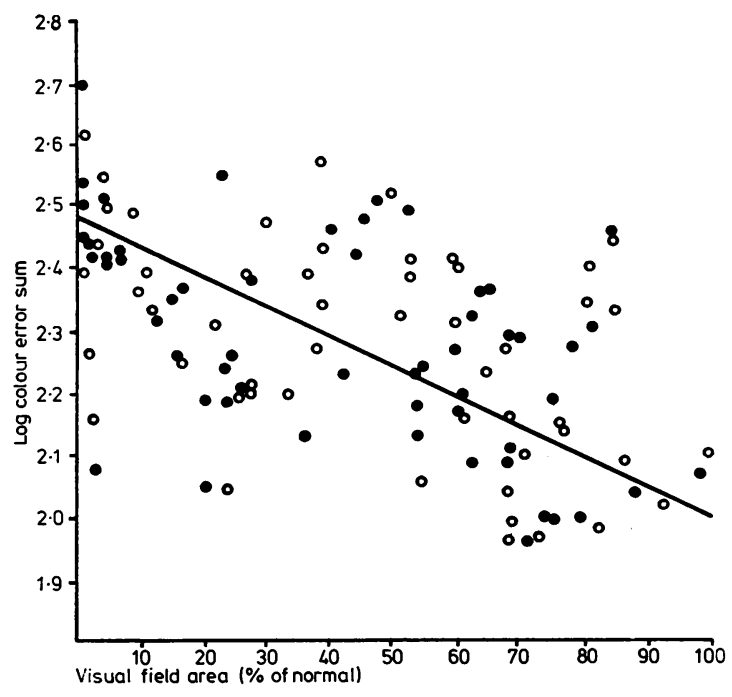

Fig. 6 Log colour error sum is plotted against percentage of normal visual field area for each patient. Closed circles represent data for the right eye and open circles represent data for the left eye. The best-fit line from a linear regression analysis is plotted along with the data points

we conclude there is symmetry in the pattern of visual function loss. The high correlation between loss of foveal colour discrimination and loss of visual field implies the existence of a bilaterally symmetrical, progressive, diffuse component to the
RP mechanism that similarly affects both foveal and peripheral visual function.

Supported by research grants from the National Institutes of Health (EY-01791) and the National Retinitis Pigmentosa Foundation.

\section{References}

Adams, A., Aspinall, P., and Hayreh, S. S. (1972). Primary retinal pigmentary degeneration. Transactions of the Ophthalmological Societies of the United Kingdom, 92, 233-249.

Berson, E. L. (1971). Light deprivation for early retinitis pigmentosa. Archives of Ophthalmology, 85, 521-529.

Biro, I. (1963). Symmetrical development of pigmentation as a specific feature of the fundus pattern in retinitis pig. mentosa. American Journal of Ophthalmology, 55, 11761179.

Carr, R. E., and Siegel, I. M. (1973). Unilateral retinitis pigmentosa. Archives of Ophthalmology, 90, 21-26.

Gonin, J. (1901). Le scotome annulaire dans la dégénérescence pigmentaire de la rétine. Annales d'Oculistique, 125, 101-130.

Jay, B. (1972). Hereditary aspects of pigmentary retinopathy. Transactions of the Ophthalmological Societies of the United Kingdom, 92, 173-178.

Pearlman, J. T. (1977). Retinitis pigmentosa: an improved clinical approach. Documenta Ophthalmologica, Proceedings of the 14th ISERG Symposium, 235-238.

Sloan, L. L. (1971). The Tübinger perimeter of Harms and Aulhorn: recommended procedures and supplementary equipment. Archives of Ophthalmology, 86, 612-622.

Sunga, R. N., and Sloan, L. L. (1967). Pigmentary degeneration of the retina: early diagnosis and natural history. Investigative Ophthalmology, 6, 309-325.

Zeavin, B. H., and Wald, G. (1956). Rod and cone vision in retinitis pigmentosa. American Journal of Ophthalmology, 42, 253-269. 